\title{
Mevalonate kinase deficiency: therapeutic targets, treatments, and outcomes
}

\section{Annalisa Marcuzzi, Elisa Piscianz, Liza Vecchi Brumatti \& Alberto Tommasini}

To cite this article: Annalisa Marcuzzi, Elisa Piscianz, Liza Vecchi Brumatti \& Alberto Tommasini (2017): Mevalonate kinase deficiency: therapeutic targets, treatments, and outcomes, Expert Opinion on Orphan Drugs, DOI: 10.1080/21678707.2017.1328308

To link to this article: http://dx.doi.org/10.1080/21678707.2017.1328308

Accepted author version posted online: 08 May 2017.

Submit your article to this journal $\pi$

View related articles $\nearrow$

View Crossmark data $₫$ 
Publisher: Taylor \& Francis

Journal: Expert Opinion on Orphan Drugs

DOI: $10.1080 / 21678707.2017 .1328308$

Mevalonate kinase deficiency: therapeutic targets, treatments, and outcomes

Annalisa Marcuzzi1, Piscianz Elisa1, Liza Vecchi Brumatti2, Alberto Tommasini2

1 Department of Medicine, Surgery, and Health Sciences, University of Trieste, Strada di Fiume,

447 Trieste, Italy. 34128 Trieste, Italy;

2 Institute for Maternal and Child Health - IRCCS "Burlo Garofolo", via dell'Istria, 65/1, 34137

Trieste, Italy.

Annalisa Marcuzzi: BSc, PhD,

Piscianz Elisa: BSc, PhD,

Liza Vecchi Brumatti: MSc

Alberto Tommasini: MD, PhD,

Author to whom correspondence should be addressed:

E-Mail: annalisa.marcuzzi@burlo.trieste.it

Tel.: +390403785422.

Article highlights.

- Mevalonate Kinase Deficiency (MKD) is a neglected disease with onset in infancy.

- MKD clinical picture is characterized by recurrent fever attacks, abdominal pain arthralgia, lymphadenopathy and, in the most severe case, neurological involvement.

- The main MKD pathogenic hypothesis is represented by a shortage of isoprenoid compounds that led to a defective geraylgeranylation of RhoA proteins and increased caspase-1-dependent inflammation.

- Mitochondrial dysfunction and altered production of 25-hydroxycholesterol represent innovative pathogenic assumptions to understand the MKD progression.

- The emerging evidences on MKD pathogenesis could widen the therapeutic approaches focused particularly on Mitochondrial-Target Antioxidants or squalene synthetase inhibitors.

\section{ABSTRACT}

Introduction: Mevalonate Kinase Deficiency (MKD) is a rare inborn disease caused by the mutation of mevalonate kinase gene. 
The clinical phenotype encompasses recurrent fever episodes in combination with gastrointestinal, immunological, rheumatological and neurological complaints. No specific treatment is available, apart from the newly approved biologics (canakinumab), but MKD can be still considered an orphan-drug disease, since the identification of a reliable therapeutic target needs an improved knowledge on the pathogenesis of the disease, which is so far controversial.

Areas covered: On one hand, shortage of isoprenoid compounds downstream of mevalonate led to a defective geranylgeranylation of RhoA/Rac proteins and increased caspase-1-dependent inflammation. On the other hand, recent studies pointed the attention to the pathogenic role of the mitochondrial dysfunction and to defective production of 25-hydroxycholesterol. These mechanisms are not exclusive of each other, as they can contribute to different pathogenic features of MKD.

Expert opinion: Innovative therapeutic approaches to MKD may count upon various medicaments, such as isoprenoid compounds that can enter the metabolic pathway, specific enzyme inhibitors and mitochondria-targeted drugs. Some of these compounds have already passed the clinical phase for other uses and may be repositioned to the treatment of MKD, fostering the development of clinical trials.

Keywords: rare disease , cholesterol , immunology, inflammation 


\section{INTRODUCTION}

Mevalonate kinase gene (MVK, 12q24.11, NM_000431) encodes for the mevalonate kinase (E.C. 2.7.1.36), a peroxisomal enzyme of the cholesterol pathway. Mutations in this gene have been found causative of a rare orphan disease, called Mevalonate Kinase Deficiency (MKD, OMIM \#260920) [1, 2].

MKD belongs to autoinflammatory syndromes, a group of inborn errors of immunity presenting with recurrent, seemingly unprovoked, multi-organ inflammatory attacks.

MKD includes different forms of disease severity ranging from the mildest Hyperimmunoglobulinemia D with periodic fever syndrome (HIDS, MIM \# 260920) to the more severe form called mevalonic aciduria (MA, OMIM \#610377), depending on the extent of the enzyme defect [3-5].

To date, MVK mutations associated with MKD are 204 and are present in almost all the exons without a "hot spot" area. The replacement c.1129 G> A (V377I) (1129G> A) is present in the majority of HIDS patients, especially in Northern Europe (> $80 \%$ ) which was shown founder effect made which would spread to the rest of Europe and USA. The V 3771 was found in homozygous in a few subjects, is usually in heterozygous combined with a second mutation associated with HIDS and / or MA phenotype [2,5].

The clinical picture of MKD, as a whole, is characterized by recurrent inflammatory attacks with fever (4-5 days duration), arthralgia, rash, abdominal pain and diarrhea with early onset in infancy, subcutaneous edema and measles-like rash. Fever rises abruptly and often above $40^{\circ} \mathrm{C}$, after which the temperature gradually returns to normal and, typically, recurrent febrile episodes diminish over the years. It can mimic other chronic disorders and thus many patients may remain undiagnosed for a long time and can be referred to a variety of subspecialty clinics, including neurology, gastroenterology, cardiology and genetics. Other clinical features have been associated with MKD in anecdotal reports or in small series, including retinitis pigmentosa, renal angiomyolipoma [6, 7] (Table 1).

The complications of the disease, occasionally present in adults, include amyloidosis [8-10], adhesions in the abdomen and, very rarely, joint contractures [11].

Patients with MA display a worse phenotype with recurrent fever attacks, developmental delay, dysmorphic features, ataxia, cerebellar atrophy. The prognosis for MA is poor: more than $50 \%$ of patients die because of an inflammatory crisis in infancy, and very few survive to adolescence [12]. During flares, acute phase reactants (ESR, CRP, and white blood cell count) and inflammatory markers (interleukin (IL)-1 $\beta$, IL-6, TNF- $\alpha$ ) are increased in peripheral blood while high levels of mevalonic acid can be measured in urines [13]. In the periods between episodes, these exams tend to be normal, except in the most severe cases and in subjects with MA. In addition, anemia mild to moderate is usually present irrespective of the phase of disease and an increase in 
transaminases and creatine phosphokinase is recorded in the most severe forms of the disease. MKD is typically associate with increased levels of $\lg D$ (and usually $\lg A$ ), that is where the name of hyperglobulinemia D syndrome comes from. However, several reports highlighted that high $\lg D$ levels are not mandatory to MKD diagnosis [14, 15].

The distinction between MA and HIDS is based on the residual enzyme activity of MK, with levels below $1 \%$ of controls in subjects with MA, while a variable enzymatic activity, estimated between 1$10 \%$, is present in patients with HIDS. Measure of MK activity in fibroblast or in peripheral blood cells from patients is of particular importance when genetic analysis of MVK detects mutations without a known correlation with phenotype. This implies that the excretion of mevalonic acid in urine may differ significantly between the two phenotypes of the syndrome, ranging from 1-56 $\mathrm{mol} / \mathrm{mol}$ creatinine (in MA) at $0.004-0.028 \mathrm{~mol} / \mathrm{mol}$ creatinine (in HIDS) [16]. Indeed, the measure of mevalonic acid in the urines collected during 24 hours of a febrile attack can serve as reliable and noninvasive diagnostic tool. Moreover, the detection of increased urinary mevalonate between febrile crises should be regarded as a score of greater severity of the disease.

The diagnosis, indeed, must be confirmed by molecular analysis of the MVK gene to establish the mutations.

\section{MKD PATHOGENESIS}

The MKD pathogenesis is not yet completely known. The finding of pathogenic MVK mutations in a subject with the typical symptoms of MKD clearly confirm support the diagnosis of MKD. However, most of patients are in well health conditions outside the inflammatory crisis and it is not clear what mechanisms trigger the inflammation. The characteristic of seemingly unprovoked bouts of inflammation dominated by high/production of the IL-1 $\beta$ allows classifying MKD among autoinflammatory disease. However, unlike other autoinflammatory diseases, MKD is not due to the mutation of genes directly involved in the control of innate inflammation. Indeed, the pathogenic mutations causative of most genetic autoinflammatory disorders involve genes codifying for components of the multiprotein platform called inflammasome. The most important and studied protein for autoinflammatory diseases is NOD-like receptor (NLR) pyrin domaincontaining 3 (P3), NLRP3 also indicated as NALP3 [17]. The NLRP3 is directly involved in activation of pro-caspase-1 to caspase-1, which in turn is required for processing the inflammatory

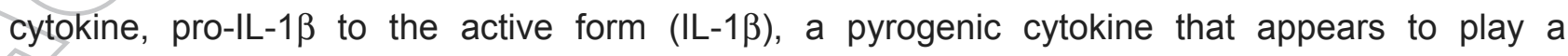
fundamental role in autoinflammatory disease with periodic fever, such as Cryopyrinopathies, Familial Mediterranean Fever, TNF Receptor Associated Periodic Fever and MKD itself [18, 19].

The production of IL-1 $\beta$ plays a crucial role in the pathogenesis as indicated by the dysregulated secretion of this inflammatory cytokine in peripheral blood cells of patients with MKD [20-22]. There is agreement that the increased production of this cytokine in MKD can be sustained both by 
activation of the transcription factor NF-kB, which modulates the expression of inflammatory genes, and by the activation of caspase-1, which is responsible for the release of the active cytokine [23]. However, according to other evidences, a defect or delay in the phagocytosis of apoptotic leukocytes can also play a role, resulting in defective suppression of inflammation [24, 25] (Figure 1).

\subsection{Mevalonate pathway in different districts of the body}

To unravel the pathogenesis of MKD and to identify the best targets for therapeutic intervention, it is fundamental to take into due consideration the different regulation of the cholesterol metabolism in the different district: systemic circle, liver, immune cells and central nervous system.

Cholesterol is supplied to the organism both by diet (exogenous lipoprotein pathway), even if it accounts for a small percentage, and by endogenous biosynthesis, mostly occurring in the liver. Mainly the liver and macrophages are involved in regulating the balance of cholesterol in the organism and in tissues. To be roughly schematic, the liver produces large amounts of cholesterol to form bile acids and to load lipoproteins. The balance between uptake and synthesis of lipoproteins in liver is crucial to the maintenance of the body lipid homeostasis. Indeed, the excess cholesterol in tissues is mainly removed through High Density Lipoproteins, which are loaded with cholesterol. Alternatively, cholesterol is removed from tissues by macrophages engulfed with dead cell membranes [26].

While liver and macrophages play a major role in the metabolism of cholesterol for most tissues, brain cells have to count only on de novo synthesis, as the blood-brain barrier effectively prevents the passage of lipoproteins. Nonetheless, brain tissues, required in high quantities of cholesterol, as it is essential for the proper functioning of the central nervous system; neurons, in fact, need cholesterol to form the membranes of axons, dendrites, and synapses [27, 28]. Moreover, there are evidences that the homeostasis of this compound is not uniform in the different brain areas: the content [29] and the expression levels of cholesterol-specific enzymes show strong variations in the different regions of the brain [30,31].

However, regulation of the mevalonate-cholesterol pathway in cell is not solely devoted to the production of cholesterol for cell membranes. Several intermediate products of this pathway have crucial roles in cell function and their regulation can vary hugely in different cells and tissues. This complexity makes it difficult to predict what effect a defective enzyme can have on the clinical phenotype. For this reason, appropriate animal and cellular models are necessary to understand the physiopathology of MKD and to study the pharmacologic intervention with compounds acting on this biochemical pathway. 


\subsection{Animal or cellular MKD models}

The possibility of using an instrument that reproduces as well as possible the inflammatory phenotype characteristic of MKD is crucial to identify molecular targets useful to develop future treatments for the disease.

\section{Genetic Model}

To date, an early issue in assessing the MKD pathogenesis is represented by the lack of a reliable genetic model. Knockout of MK in animals is not compatible with life, highlighting the importance of cholesterol and intermediate compounds for several vital cell functions. Unfortunately, in mice, the homozygous knock-in of mutations associated with human MKD was also unsuccessful. Thus, the only genetic mouse model of MKD has been obtained by the heterozygous deletion of one MVK allele; however, this model only partially reproduces the MKD-like inflammatory phenotype and does not show the features of neurological dysfunction [32].

Similarly, there have been difficulties in developing an in vitro genetic model. Recently a report showed that the knockdown of mevalonate kinase, using the silencing RNA (siRNA) technology, in a murine microglial cellular model (BV-2 cells) is not a suitable MKD model, because the NLRP3 expression and the apoptosis does not correspond to the expression obtained from MKD patients [33].

\section{Biochemical Model}

To overcome these limitations, in recent years, our and other groups have set an MKD model, both in vitro and in vivo, using pharmacological inhibitors to induce a biochemical imbalance mimicking the genetic deficiency of MK. Indeed, compounds such as statins or amino-bisphosphonates block the synthetic pathway of cholesterol and provoke an inflammatory dysregulation similar to that characteristic of MKD. On this substrate, the inflammatory attacks were triggered by the addition of a bacterial compound (lipopolysaccharide or muramyl-dipeptide) stimulating natural immunity activation. In this way, this model tried to reproduce the condition of patients with MKD, where the febrile attacks are often associated with minor external stimuli such as vaccination, infection or physical exertion [34].

The results obtained from both monocytes and neuronal cell lines treated with such inhibitors show that the shortage of intermediate compounds downstream of mevalonate can induce an inflammatory phenotype. Using the same experimental approach, mice with impaired biosynthesis of mevalonate-derived isoprenoid, displayed an increased inflammatory reactivity to the inflammatory stimulus muramyl-dipeptide [35]. This model appears to be particularly consistent with MKD, since it reproduces the molecular markers typical of the disease in humans (SAA Serum 
amyloid A, inflammasome activation, biosynthesis of inflammatory cytokines), regardless of the mouse strain used [36].

The availability of these models, even with their weaknesses and limitations arising from a biochemical system, offers a valuable opportunity to address the study of the pathogenesis of MKD.

This is particularly important to investigate the neurological features in MA [37, 38]. In fact, whilst peripheral blood cells obtained from patients can allow studies relevant to systemic inflammation, no neurological tissues can obviously be available for ex vivo researches.

Moreover, the establishment of accurate in vitro and in vivo models for MA can represent a first step towards a better understanding the neuroinflammatory events occurring not only in MA, but also in other neurodegenerative disorders in which the cholesterol pathway is involved, including Alzheimer's disease, Huntington's disease, Parkinson's disease, Niemann-Pick type C disease and Smith-Lemli Opitz syndrome [39].

\subsection{Shortage of isoprenoids underlies MKD inflammation}

The defects in MK, logically could suggest that an accumulation of upstream intermediates, like mevalonate, occurs in MKD and related diseases and that this accumulation could be responsible for the pathological features of the diseases. On the contrary, the experiences with statins and the evidences that these drugs can block the pathway without resulting in inflammatory processes, suggest that the pathogenesis arises likely from a shortage of downstream products, rather than the accumulation of upstream intermediates [40].

Indeed, it was shown that the decrease of the intermediate isoprenoid levels, is responsible for the increased secretion of inflammatory cytokine IL-1 in MKD [21]. However, literature data demonstrated that even though MK enzyme activity is reduced and the residual MK levels are below detection levels in cultured skin fibroblasts from MA patients, the de novo biosynthesis of cholesterol and non-sterol isoprenoids can be sufficient [40,41]. This paradox is probably due to the compensatory induction of 3-hydroxy-3-methylglutaryl coenzyme A (HMG-CoA) reductase leading to increased concentration of mevalonate. Indeed, the increased substrate of MK may allow the production of sufficient amount of the product [41, 42]. However, albeit sufficient in most physiological condition, this mechanism may be overcome in stress conditions, when the organism may needs higher synthesis of mevalonate-derived isoprenoids and cholesterol. Several studies highlight that the inflammatory phenotype associated with MKD is attributable to the inability of this system to compensate an instant further decrease in the MK residual activity or an increased usage of some isoprenoid compound. For example, it was suggested that the regulation of the immune response may require and extra-use of isoprenoid intermediates [18, 43]. 


\subsection{Crucial role of defective geranylgeranylation of Rho and Rac in inflammation}

The complex mechanism activated by mevalonate block and supported by NLRP3 implies a shortage of isoprenoids and in particular non-sterols isoprenoids, such as geranylgeranyl-PP (GGPP). Isoprenylation with GGPP modulates the function of some membrane-bound proteins involved in the control of inflammatory response, proliferation, and apoptosis, such as the small GTPases of the Ras homolog gene family (RhoA) [44, 45]. Thus, depletion of GGPP can lead to impaired prenylation of such GTPases, resulting in an unbalanced control of inflammation. Furthermore, isoprenoids and sterols derived from mevalonate can also play a direct role in DNA transcription, enzymatic regulation and response to oxidative stress [46-48].

Notably, environmental factors can act on this scenario in different ways: nutritional factors can influence the availability of substrates and the regulation of various enzymes; immune stimuli such as infections and vaccinations can trigger an inflammatory response that involves several prenylated proteins [49]; oxidative stress of various natures can exceed the antioxidant capacity of a defective mevalonate pathway $[50,51]$.

\subsection{Mitochondrial dysfunction in the pathogenesis of inflammation}

Recent evidences of the literature point out that to understand the pathogenesis of MKD is necessary to focus on the role of the mevalonate pathway on the modulation of autophagy.

Autophagy can be triggered by mitochondrial dysfunctions, when the energy produced is reduced and the cell activates a saving mode to preserve its integrity. This program can lead to reduced performances of the cells, metabolism, and reuse of macromolecules and digestion of damaged mitochondria.

Recent literature evidence emphasizes that mitochondrial damage and induction of apoptosis can be due to various intracellular stress conditions, including DNA damage, cytotoxic insult, oxidative stress, infections. These various stimuli act by inhibiting or activating a particular anti- and proapoptotic Bcl-2 family member [52]. The Bcl-2-associated X protein (Bax) is known to be a very important pro-apoptotic protein promoting the formation of channels into the mitochondria. The Bax-induced channels formed in the mitochondria are important for the dissipation of the mitochondrial transmembrane potential $(\Delta \psi \mathrm{m})$, the inhibition of the respiratory chain and the interruption of the production of reactive oxygen species (ROS) [53]. Similar mitochondrial dysfunctions also represent a central causal factor in the pathogenesis of neurodegenerative diseases such as Alzheimer's disease, Parkinson's disease, Huntington's disease and many others [54].

Recent studies on the action of genistein, an isoflavonoid compound acting on the mevalonate pathway, established a link between this pathway and oxidative stress in neurons. In particular, 
genistein is able to recover the ROS production, to favor an anti-apoptotic Bcl-2/Bax ratio and contrast the NF-kB and MAPK inflammatory pathways. The potential neuroprotective and antinflammatory action of this natural compound may indirectly support the role of mitochondrial stress in MA [55].

\subsection{Defective 25-hydroxycholesterol may underlie hyperimmunoglobulinemia D and inflammation}

About 10 years ago, a pioneering work of Goldstein [56] described the hydrophobic molecule 25hydroxycholesterol $(25-\mathrm{HC})$ within the metabolic pathway of cholesterol. Since then, many studies have followed with the aim of establishing a biological role for this molecule [57-59]. In particular, $25-\mathrm{HC}$ is a lipid with immunoregulatory potential, produced by macrophages to downregulate the adaptive immune response [60]. Of note, the lack of $25-\mathrm{HC}$ can be responsible for an increased production of Immunoglobulin A, which is typical of the most severe forms of MKD (such as MA) (Table 1). Furthermore, other studies showed that 25-HC could suppress the activation of NLRP3 and consequently the production of inflammatory cytokines such as IL-1 [60]. Based on this data, it could be logical to hypothesize that a shortage of $25-\mathrm{HC}$ may play a role in the pathogenesis of inflammation and hyperimmunoglobulinemia in MKD [61]. However, recent data showed that 25$\mathrm{HC}$ could have as well inflammatory effects, as in the case of cerebral inflammation associated with X-linked adrenoleukodystrophy [62]. Thus, the role of 25-HC in MKD remains controversial.

\section{DRUGS ACTING ON THE MEVALONATE PATHWAY AND POTENTIAL ROLE IN MKD}

Drugs acting on the mevalonate pathway may have variable effects on inflammation, with sometimes-unpredictable consequences in relation to dosage. In fact, the enzymes in the mevalonate pathway are highly regulated according to the availability of substrates and products. Such drugs have the ability to interfere with the metabolic pathway and are described in the following paragraphs (3.1 to 3.5), while in Chapter 4 actual and future therapeutic options for MKD will be discussed.

\subsection{Statins}

Statins, also known as HMG-CoA-reductase inhibitors, are a class of lipid-lowering drugs, acting on the rate-limiting step in the cholesterol pathway. The statins, indeed, act upstream of the mevalonate pathway by reducing the availability of mevalonate and the production of endogenous cholesterol [63-66]. 
Statins have been considered to treat MKD, with the aim of inducing a compensatory effect on the residual mevalonate kinase activity. In a small group of HIDS patients, continuous treatment with simvastatin seemed associated with a lowered number of inflammatory crisis [67]. Nevertheless, the treatment with lovastatin in patients with MA led to a severe worsening of the clinical symptoms [68]. Taken together, these data suggest that statins can allow some improvement in subjects with milder diseases, like HIDS phenotype, while they will worsen the defect in subjects with more severe deficiency, such as MA. Thus, the use of statins in MKD remains controversial. Of note, several studies showed that statins could exert either anti- or pro-inflammatory effects in slightly different conditions [69].

\subsection{Amino-bisphosphonates}

The amino-bisphosphonates are nitrogenous bisphosphonates with a strong effect in preventing bone resorption. Thanks to their chemical structure with two phosphate groups, these drugs are highly concentrated in bones where they act by inhibiting the function of osteoclasts. The high concentration of the drug reached in bones makes the amino-bisphosphonates particularly useful to prevent and treat osteoporosis, but also to contrast bone inflammation or bone metastasis. In particular, the amino-bisphosphonates such as alendronate, pamidronate, zoledronate, act on farnesyl diphosphate synthase (FPPS), just downstream of mevalonate kinase and or on other enzymes in the pathway to cholesterol, reducing in a variable manner the availability of isoprenoid intermediates. Inhibition of mevalonate pathway enzymes leads to various biological effects, including inhibition of osteoclast on tumor metastatic cells [70-72]. Alike occurs in MKD, the action of the amino-bisphosphonates leads to an accumulation of mevalonate and at the same time the reduction of downstream compounds of FPPS.

Amino-bisphosphonates can be either anti- or pro-inflammatory, depending on the experimental context, similarly to what occurs for statins $[73,74]$. The different effects may be related to their different ability to be metabolized by the cells [75].

\subsection{Plant-derived isoprenoids}

Isoprene (also called isoprene units) is the common name for 2-methyl-1,3-butadiene, a compound that contains five carbon atoms, characterized by a branched "head" and a "tail". These ends of each isoprene unit can be joined "head-to-head", "head-to-tail" or "tail-to-tail" to form different terpenes. Terpenes are a class of lipids: they can be hydrocarbons or alcohols containing oxygen, aldehydes or ketones.

To date, there are more than 20,000 known terpenes. Many plants, particularly conifers, produce a huge variety of them and some insects. Terpenes are the main components of resins and essential 
oils of plants, mixtures of substances that give each flower or plant a distinctive odor or flavor. They, also, are the biosynthetic precursors of steroids and cholesterol. Many flavorings used in foods and fragrances were derived from natural terpenes or terpenoids, as well as many perfumes and flavors found in plants and known as essential oils.

Plant Moreover, isoprenoids are can be found as intermediate compounds of the mevalonate pathway, so that while some of the plant isoprenoids can enter the metabolic pathway in humans (i.e. geraniol, farnesol, geranylgeraniol, etc.), or can act as enzyme inhibitors (i.e. menthol, limonene, etc).

The geraniol $(\mathrm{GOH})$ for example, is a terpene alcohol extracted from certain types of plant (geranium, orange, vervain, myrtle, lemon balm) and commonly used in perfumery and food industry. The geraniol, relatively abundant in nature, is readily soluble in alcohol and in ether, with excellent good chemical stability and fat solubility, appearance that makes it ideal for a good absorption through different routes of administration. Some authors have already described the use of geraniol for topical use [76] or as an adjunct to anti-cancer drugs [77]. Likewise, menthol $(\mathrm{MOH})$ is also a monoterpene, the active ingredient in the essential oil content of mint. Strongly aromatic and bitter, menthol is particularly known for its antiseptic, antispasmodic and local anesthetic action. The inhalation of the vapors that developed by placing a few drops of alcoholic solution of menthol in boiling water is famous for its ability to relieve the bronchial disorders and nasal congestion; menthol in fact can act as expectorant and disinfectant. Taken orally, the menthol is also used as anti-fermentative, antiseptic (facilitates the excretion of bile and runoff), refreshing and spasmolytic (reduces intestinal motility), which is useful in the presence of colics of the upper gastrointestinal tract, biliary tract and colon irritations, such as irritable bowel syndrome $[78,79]$.

It What is also very interesting from the medical aspect is the antinflammatory properties highlighted in studies on geranylgeraniol (GGOH), a diterpene soluble in organic solvents such as alcohol and acetone.

Moreover, some of these compounds are already used as adjuvants in cancer therapies and as anti-metastatic agents [77, 80].

\subsection{Inhibitors of prenylation}

Prenylation inhibitors act individually on the enzymes farnesyltransferase (FT) and geranylgeranyltransferase (GGT), which catalyze the transfer of isoprenoid "activated" groups (FPP or GGPP) on polypeptide chains.

The characterization and the role of farnesylation and geranylgeranylation protein have been made possible by using specific inhibitors: inhibitors of farnesyltransferase (FTI) and geranylgeranyltransferase (GGTI). 
The FTI act on different mechanisms of inhibition: some of them, such as manumycin A (ManA), a natural compound derived from Streptomyces, belong to the class of isoprenoid derivatives, in this case of the FPP analogs, while others, to the class of peptidomimetic.

The inhibitors of GGT act similarly to FTI as competitors. The discovery that some geranylgeranylated proteins are involved in pathological processes such as cancer, inflammation, and viral infections; has led to a growing interest in the study of GGT as a possible therapeutic targets. They have been described several peptidomimetic GGTI, such as GGTI-298 and GGTI2154 , both amino benzoic acid derivatives [81]. Recently it has been observed that some inhibitors belonging to the class of FTI can also inhibit GGT-type 2, also known as Rab-GGT since it is specific for Rab proteins [82].

The RAS family of proteins must be prenylated to properly perform their function and therefore are the main targets of such inhibitors. In particular, the FTI can inhibit RAS, while the GGTI-type 1 inhibit Rho and Rac [82, 83].

\subsection{Zaragozic acid}

Noteworthy is the effect of the zaragozic acid (ZAA), an inhibitor of squalene synthase. ZAA can reduce the synthesis of cholesterol, redirecting the flux throughout the cholesterol pathway to isoprenoids biosynthesis and functioning as a potential new cholesterol lowering agent [83-85].

ZAA is an inhibition of the competitive type, because it mimics the enzyme substrate presqualene$\mathrm{PP}$, which typically forms during the reaction leading to the synthesis of squalene [86].

Recently ZAA has been proven to inhibit the hyper-inflammatory response in cellular models of MKD, by diverting mevalonate intermediates toward geranylgeranylation rather than to the synthesis of squalene.

\section{PHARMACOLOGIC OPTIONS IN MKD}

Although in recent years treatments for these rare diseases have been developed, it is worthy highlighting that, to date, there is no pathway-oriented treatment available for MKD. Indeed, current options are primarily intended to prevent or alleviate inflammatory symptoms, while future pharmacological treatments have the goal to be more close to the pathogenesis of the disease and more effective for patients with severe MKD (Figure 2).

\subsection{Current MKD treatments and biological drugs}

Several drugs have been used in MKD, but few of them still maintain a role in the treatment of the disease. In particular, the disease usually shows a good response to corticosteroids, but the adverse effects of these drugs can soon overcome the benefits, in particular when there is a need 
for prolonged treatment. The major side effects associated with the traditional MKD treatments are a headache, stomach ulcers and kidney damage [4].

While antinflammatory drugs and steroids on demand provide acceptable control of symptoms in patients with milder forms of the disease, lifelong treatment with biological drugs (such as Anakinra or Canakinumab) [87, 88] is usually required for patients with high recurrence of severe inflammatory attacks. The response to anti-IL-1 biologics confirms once more the autoinflammatory nature of MKD.

Anakinra is a recombinant, non-glycosylated, IL-1Ra; an endogenous antagonist that binds to VL- 1 receptors and inhibits the inflammatory effects of $\mathrm{IL}-1$ : it is administered subcutaneously and present a high bioavailability (95\%). Other anti-IL-1 biologics include Rilonacept, a fully human fusion protein that incorporates the extracellular domains of the IL-1 receptor linked to the Fc portion of IgG1 and Canakinumab, a monoclonal antibody specific for $\mathbb{L}-1 \beta$. Canakinumab, approved both in Europe and USA, has been studied for the treatment of rheumatologic diseases such as rheumatoid arthritis and systemic juvenile idiopathic arthritis, and for autoinflammatory diseases such as cryopyrin-associated periodic syndrome (CAPS). Very recently, Canakinumab has been approved by US Food and Drug Administration (September 2016) and by European Medicines Agency (December 2016) as treatment for MKD after the substantial results obtained in a Phase III Study (www.clinicaltrials.gov, NCT02059291).

These biologicals have been shown effective in controlling MKD-related inflammation in clinical reports. However, the use of Anakinra is hindered by the need of daily painful injections, even if some patients well tolerate this treatment and then get good improvements [65,66]. Canakinumab seem to be more effective, but it is not always able to completely control the disease, in particular in cases with severe disease. Moreover, biologics, especially Canakinumab, are very expensive and, at today, the dosing is still a matter of investigation. To date, the only valuable therapeutic option for patients with MA is hematopoietic stem cell transplantation, which however is burdened with a series of risks and complications [89, 90].

\subsection{Future directions}

The aim of the innovative pharmacological strategies is to obtain a more complete control of the disease than what can be achieved with cytokine inhibitors. An ideal causal therapy is expected to control all the different signs of the disease, including the neurologic and the lymphoproliferative signs, which are not completely targeted by current treatments. Furthermore, anti-cytokine biologicals have just a theoretical risk of infections: on a particular ground it is recommended to pay attention to the risk of the infection in patients treated with anti-IL1, and this may make difficult to discriminate if the inflammatory events during the therapy are due to this is flare of infections. 
The easier therapeutic approach would be to exploit the supplementation with plant isoprenoids that can enter the mevalonate pathway compensating for the shortage of endogenous isoprenoids. This approach is based on the experimental evidence of the role of the shortage of isoprenoids in MKD inflammation. Moreover, several plant isoprenoids have been reported to have antinflammatory properties and could represent an attractive therapeutic opportunity thanks to their commercial availability, low cost, oral bioavailability and low toxicity. Moreover, recent literature data have showed that these natural products can also regulate in central nervous system, in particular, controlling motor and cognitive function [91].

Nevertheless, a limit to the translational of plant isoprenoids to the therapeutics of MKD can be represented by the amount of substance needed to have the antinflammatory benefit in vivo, which could be above the tolerated doses.

Another approach is based on the use of inhibitor enzymes that can diverge the flux of isoprenoids toward antinflammatory molecules. This is the case for FTls, like the such as Tipifarnib (R115777, Zarnestra $^{\mathrm{TM}}$ ) and Lonafarnib ( $\mathrm{SCH} 66336$, Sarasar ${ }^{\mathrm{TM}}$ ), which showed their potential use as antiparasitic, antifungal, antiviral and anticancer like, thanks to their effects exerted on oncoproteins such as RAS [92]. Preliminary in vitro studies on MKD monocytes treated with Tipifarnib and geraniol showed a significative reduction of $I L-1 \beta$ production, supporting the idea that an effective therapy for MKD may need combinatory approaches.

Another attractive therapeutic possibility is represented by Lapaquistat (TAK-475, Takeda) [93, 94], an analogue inhibitor of zaragozic acid, a squalene synthetase inhibitor, which is a drug that underwent extensive clinical trials as cholesterol lowering agent 10 years ago with a good safety profile [95-98]. The possibile advantages for this compound are that it has already passed all the clinical stages and that could be evaluated in clinical trials and it can be administerly orally.

The potential use of Lapaquistat seem to be in contrast with data showing a role for defective 25$\mathrm{HC}$ in MKD, as squalene synthase inhibition is expected to lower even more the levels of $25-\mathrm{HC}$ [34]. However, the role of $25-\mathrm{HC}$ remains controversial, as in adrenoleukodystrophy this molecule seem to be responsible for activation of NLRP3 and neuroinflammation [62]. Due to the complex regulation of the mevalonate pathway, only pre-clinical studies in animals and pilot studies in human will clarify this controversy indicating the correct direction for innovative treatments of MKD. Drugs belonging to the Mitochondrial-Target Anti-oxidants (MTAs) family, such as MitoQ, Mitotempo, represent as well a promising pharmacological approach and MitoVitE could potentially be effective in tackling the disease. MTAs, which are well known and well characterized for their anti-oxidant and mitochondrial protective activity in response to Bax, could indeed be potential candidate molecules to improve MA treatment. Moreover, MTAs are mitochondria-targeted drugs, several hundred folds more potent at in preventing mitochondrial oxidative damage than an 
untargeted antioxidant [99] and, once accumulated into mitochondria, MTAs are able to inhibit step by step the activation of programmed cell death pathways [100].

\section{EXPERT OPINION}

MKD is still a rare orphan drug disease with onset in infancy. Only drugs able to reduce the inflammatory symptoms are used in clinical practice, often with incomplete control of symptoms. Thus, patients with severe MKD still tend to have a poor quality of life.

Patients with mevalonic aciduria, indeed, have high morbidity and reduced life expectancy in spite of current treatments. In some cases, while attenuating the flares, the treatments (especially with glucocorticoids) can lead to a chronic course of disease with weaker but constant symptoms.

Furthermore, recent data indicate that MKD often remains misdiagnosed for years and the burden of MKD may be even greater than expected. Thus, the development of an effective drug to cure classical MKD might provide novel options for a larger number of patients than those suffering from diagnosed MKD.

Moreover, the development of new models, both in vitro and in vivo, that can reproduce the neurological involvement in MA, is crucial to improve the knowledge on its pathogenesis, which is still unknown, and to develop new therapies able to improve the pathological conditions of patients. The dietary approaches have not yet considered. Even if MKD may be associated with low cholesterol, reducing the intake of cholesterol with diet may have a beneficial effect in MKD, by inducing an upregulation of the endogenous biosynthetic machinery.

In general, it is important to emphasize that MKD belongs to the family of the autoinflammatory syndromes (AIS), a group of rare disorders, often underestimated or misdiagnosed and characterized by difficult therapeutic issues.

From a scientific point of view, the AIS provide a clear model to understand the mechanisms involved in controlling inflammation and to study how combined mutations in functionally related genes may condition these pathologies as well as more common inflammatory disorders.

Furthermore, in-depth knowledge on autoinflammatory disease may allow identifying novel targets for antinflammatory therapies, both for AIS and AIS-like disorders. In particular, studying the potential impact of manipulation of the mevalonate pathway on inflammation may open novel avenues for antinflammatory therapies. The identification, also, of epigenetic signatures associated with the development of AIS in genetically predisposed individuals may provide new tools to trace how environment and medical treatments influence the course of the disease.

\section{Funding}

This paper was funded by the ministry of Health grant RC 36/11

Declaration of Interest 
T Alberto performed experimental research for Takeda in 2014 receiving a total amount 18.000 euros for research expense. The authors have no other relevant affiliations or financial involvement with any organization or entity with a financial interest in or financial conflict with the subject matter or materials discussed in the manuscript apart from those disclosed. 


\section{REFERENCES}

Papers of special note have been highlighted as:

* of interest

** of considerable interest

1. Favier LA, Schulert GS. Mevalonate kinase deficiency: current perspectives. Appl Clin Genet 2016;9:101-10.

2. Ter Haar NM, Jeyaratnam J, Lachmann HJ, et al. The Phenotype and Genotype of Mevalonate Kinase Deficiency: A Series of 114 Cases From the Eurofever Registry. Arthritis Rheumatol 2016;68:2795-805.

3. Drenth JP, Haagsma CJ, van der Meer JW. Hyperimmunoglobulinemia $D$ and periodic fever syndrome. The clinical spectrum in a series of 50 patients. International Hyper-lgD Study Group. Medicine (Baltimore) 1994;73:133-44.

* The first paper describing a rigorous multicentre study on MKD.

4. van der Hilst JC, Bodar EJ, Barron KS, et al. Long-term follow-up, clinical features, and quality of life in a series of 103 patients with hyperimmunoglobulinemia D syndrome, Medicine (Baltimore) 2008;87:301-10.

5. Stabile A, Compagnone A, Napodano S, et al. Mevalonate kinase genotype in children with recurrent fevers and high serum IgD level. Rheumatol Int 2013;33:3039-42.

6. Balgobind B, Wittebol-Post D, Frenkel J. Retinitis pigmentosa in mevalonate kinase deficiency. J Inherit Metab Dis 2005;28:1143-5.

7. Kellner U, Stöhr H, Weinitz S, et al. Mevalonate kinase deficiency associated with ataxia and retinitis pigmentosa in two brothers with MVK gene mutations. Ophthalmic Genet 2017:1-5.

8. Kallianidis AF, Ray A, Goudkade D, de Fijter JW. Amyloid A amyloidosis secondary to hyper IgD syndrome and response to IL-1 blockage therapy. Neth J Med 2016;74:43-6.

9. Yel S, Gunduz Z, Bastug $F$, et al. Amyloidosis in a child with hyperimmunoglobulinemia D syndrome. Iran J Kidney Dis 2013,7:70-2.

10. Lane T, Loeffler JM, Rowczenio DM, et al. AA amyloidosis complicating the hereditary periodic fever syndromes. Arthritis Rheum 2013;65:1116-21.

11. Zhang S. Natural history of mevalonate kinase deficiency: a literature review. Pediatr Rheumatol Online J 2016;14:30.

12. Haas D, Hoffmann GF. Mevalonate kinase deficiencies: from mevalonic aciduria to hyperimmunoglobulinemia D syndrome. Orphanet J Rare Dis 2006;1:13.

13. Jeyaratnam J, Ter Haar NM, de Sain-van der Velden MG, et al. Diagnostic Value of Urinary Mevalonic Acid Excretion in Patients with a Clinical Suspicion of Mevalonate Kinase Deficiency (MKD). JIMD Rep 2016;27:33-8.

14. Mulders-Manders CM, Simon A. Hyper-lgD syndrome/mevalonate kinase deficiency: what is new? Semin Immunopathol 2015;37:371-6.

15. Overed-Sayer CL, Mosedale DE, Goodall M, Grainger DJ. Measurement of human serum IgD levels. Curr Protoc Immunol 2009; Chapter 2:Unit 2.9B.

16. Hoffmann G, Gibson KM, Brandt IK, et al. Mevalonic aciduria--an inborn error of cholesterol and nonsterol isoprene biosynthesis. N Engl J Med 1986;314:1610-4.

*A pioneering paper dedicated to severe form of MKD called Mevalonic Aciduria 
17. Jo EK, Kim JK, Shin DM, Sasakawa C. Molecular mechanisms regulating NLRP3 inflammasome activation. Cell Mol Immunol 2016;13:148-59.

18. Akula MK, Shi M, Jiang Z, et al. Control of the innate immune response by the mevalonate pathway. Nat Immunol 2016;17:922-9.

19. van der Burgh R, Nijhuis L, Pervolaraki K, et al. Defects in mitochondrial clearance predispose human monocytes to interleukin-1 $\beta$ hypersecretion. J Biol Chem 2014;289:5000-12.

20. Frenkel J, Rijkers GT, Mandey SH, et al. Lack of isoprenoid products raises ex vivo interleukin-1beta secretion in hyperimmunoglobulinemia $D$ and periodic fever syndrome. Arthritis Rheum 2002;46:2794-803.

\section{${ }^{* *}$ A crucial paper describing important evidence on MKD pathogenesis}

21. Mandey SH, Kuijk LM, Frenkel J, Waterham HR. A role for geranylgeranylation in interleukin1 beta secretion. Arthritis Rheum 2006;54:3690-5.

22. Drenth JP, van Deuren M, van der Ven-Jongekrijg J, et al. Cytokine activation during attacks of the hyperimmunoglobulinemia D and periodic fever syndrome, Blood 1995;85:3586-93

23. Meyer-Hoffert $U$. Neutrophil-derived serine proteases modulate innate immune responses. Front Biosci (Landmark Ed) 2009;14:3409-18.

24. Weigert $A$, Jennewein $C$, Brüne $B$. The liaison between apoptotic cells and macrophages--the end programs the beginning. Biol Chem 2009;390:379-90.

25. Stoffels $M$, Jongekrijg J, Remijn $T$, et al. TLR2/TLR4-dependent exaggerated cytokine production in hyperimmunoglobulinaemia $D$ and periodic fever syndrome, Rheumatology (Oxford) 2015;54:363-8.

26. Fernandez-Ruiz I, Puchalska P, Narasimhulu CA, et al. Differential lipid metabolism in monocytes and macrophages: influence of cholesterol loading. J Lipid Res 2016;57:574-86.

27. Benarroch EE. Brain cholesterol metabolism and neurologic disease. Neurology 2008;71:1368-73.

28. Bifulco M. Molecular mechanisms responsible for neuroinflammation and neurological impairments in mevalonate kinase deficiency. Mol Genet Metab Rep 2015;3:42.

29. Zhang J, Liu Q. Cholesterol metabolism and homeostasis in the brain. Protein Cell 2015;6:254-64.

30. Cartocci V, Servadio M, Trezza V, Pallottini V. Can Cholesterol Metabolism Modulation Affect Brain Function and Behavior? J Cell Physiol 2017;232:281-86.

31. Bae SH, Lee JN, Fitzky BU, et al. Cholesterol biosynthesis from lanosterol. Molecular cloning, tissue distribution, expression, chromosomal localization, and regulation of rat 7dehydrocholesterol reductase, a Smith-Lemli-Opitz syndrome-related protein. J Biol Chem 1999;274:14624-31.

32. Hager EJ, Tse HM, Piganelli JD, et al. Deletion of a single mevalonate kinase (Mvk) allele yields a murine model of hyper-IgD syndrome. J Inherit Metab Dis 2007;30:888-95.

33. Celsi F, Piscianz E, Romano M, Crovella S. Knockdown of MVK does not lead to changes in NALP3 expression or activation. J Inflamm (Lond) 2015;12:7.

34. Simon A. Cholesterol metabolism and immunity. N Engl J Med 2014 Nov;371(20):1933-5.

35. Marcuzzi A, Pontillo A, De Leo $L$, et al. Natural isoprenoids are able to reduce inflammation in a mouse model of mevalonate kinase deficiency. Pediatr Res 2008;64:177-82.

36. Marcuzzi A, Zanin V, Kleiner G, et al. Mouse model of mevalonate kinase deficiency: comparison of cytokine and chemokine profile with that of human patients. Pediatr Res 2013;74:266-71.

37. Tricarico PM, Piscianz E, Monasta L, et al. Microglia activation and interaction with neuronal cells in a biochemical model of mevalonate kinase deficiency. Apoptosis 2015;20:1048-55. 
38. Marcuzzi A, Piscianz E, Zweyer M, et al. Geranylgeraniol and Neurological Impairment: Involvement of Apoptosis and Mitochondrial Morphology. Int J Mol Sci 2016;17:365.

39. Platt FM, Wassif C, Colaco A, et al. Disorders of cholesterol metabolism and their unanticipated convergent mechanisms of disease. Annu Rev Genomics Hum Genet 2014;15:17394.

40. Houten SM, Schneiders MS, Wanders RJ, Waterham HR. Regulation of isoprenoid/cholesterol biosynthesis in cells from mevalonate kinase-deficient patients. J Biol Chem 2003;278:5736-43.

41. Waterham HR. Inherited disorders of cholesterol biosynthesis. Clin Genet 2002;61:393-403.

42. Houten SM, Koster J, Romeijn GJ, et al. Organization of the mevalonate kinase (MVK) gene and identification of novel mutations causing mevalonic aciduria and hyperimmunoglobulinaemia $D$ and periodic fever syndrome. Eur J Hum Genet 2001;9:253-9.

43. Muller AL, Freed DH. Basic and Clinical Observations of Mevalonate Depletion on the Mevalonate Signaling Pathway. Curr Mol Pharmacol 2017;10:6-12.

44. van der Burgh $\mathrm{R}$, Pervolaraki $\mathrm{K}$, Turkenburg $\mathrm{M}$, et al. Unprenylated RhoA contributes to IL-1 $\beta$ hypersecretion in mevalonate kinase deficiency model through stimulation of Rac1 activity. $J$ Biol Chem 2014;289:27757-65.

45. Henneman L, Schneiders MS, Turkenburg M, Waterham HR. Compromized geranylgeranylation of RhoA and Rac1 in mevalonate kinase deficiency. J Inherit Metab Dis 2010;33:625-32.

46. Agabiti SS, Liang Y, Wiemer AJ. Molecular mechanisms linking geranylgeranyl diphosphate synthase to cell survival and proliferation. Mol Membr Biol 2016:33:1-11.

47. Jurczyluk J, Munoz MA, Skinner OP, et al. Mevalonate kinase deficiency leads to decreased prenylation of Rab GTPases. Immunol Cell Biol 2016;94:994-99.

48. Hashemi M, Hoshyar R, Ande SR, et al. Mevalonate Cascade and its Regulation in Cholesterol Metabolism in Different Tissues in Health and Disease. Curr Mol Pharmacol 2017; 10:13-26.

**A paper highlighting the crucial involvement of RhoA in MKD pathogenesis

49. Bodar EJ, van der Hilst JC Drenth JP, et al. Effect of etanercept and anakinra on inflammatory attacks in the hyper-lgD syndrome: introducing a vaccination provocation model. Neth J Med 2005;63:260-4.

50. Marcuzzi A, Piscianz E, Valencic E, et al. To Extinguish the Fire from Outside the Cell or to Shutdown the Gas Valve Inside? Novel Trends in Anti-Inflammatory Therapies. Int J Mol Sci 2015;16:21277-93.

51. Liao $P$, Hemmerlin A, Bach TJ, Chye ML. The potential of the mevalonate pathway for enhanced isoprenoid production. Biotechnol Adv 2016 2016;34:697-713.

52. Galluzzi L, Vitale I, Abrams JM, et al. Molecular definitions of cell death subroutines: recommendations of the Nomenclature Committee on Cell Death 2012. Cell Death Differ 2012;19:107-20.

53. Simon HU, Haj-Yehia A, Levi-Schaffer F. Role of reactive oxygen species (ROS) in apoptosis induction. Apoptosis 2000;5:415-8.

54. Johri A, Beal MF. Mitochondrial dysfunction in neurodegenerative diseases. J Pharmacol Exp Ther 2012;342:619-30.

55. Qian $\mathrm{Y}, \mathrm{Cao} \mathrm{L}$, Guan $\mathrm{T}$, et al. Protection by genistein on cortical neurons against oxidative stress injury via inhibition of NF-kappaB, JNK and ERK signaling pathway. Pharm Biol 2015;53:1124-32. 
56. Goldstein JL, DeBose-Boyd RA, Brown MS. Protein sensors for membrane sterols. Cell 2006;124:35-46.

57. Lappano R, Recchia AG, De Francesco EM, et al. The cholesterol metabolite 25hydroxycholesterol activates estrogen receptor a-mediated signaling in cancer cells and in cardiomyocytes. PLoS One 2011;6:e16631.

58. Olsen BN, Schlesinger PH, Ory DS, Baker NA. 25-Hydroxycholesterol increases the availability of cholesterol in phospholipid membranes. Biophys J 2011;100:948-56.

59. Diczfalusy $U$. On the formation and possible biological role of 25-hydroxycholesterol. Biochimie 2013;95:455-60.

60. Bauman DR, Bitmansour AD, McDonald JG, et al. 25-Hydroxycholesterol secreted by macrophages in response to Toll-like receptor activation suppresses immunoglobulin A production. Proc Natl Acad Sci U S A 2009;106:16764-9.

61. Cyster JG, Dang EV, Reboldi A, Yi T. 25-Hydroxycholesterols in innate and adaptive immunity. Nat Rev Immunol 2014;14:731-43.

62. Jang J, Park S, Jin Hur H, et al. 25-hydroxycholesterol contributes to cerebral inflammation of X-linked adrenoleukodystrophy through activation of the NLRP3 inflammasome. Nat Commun 2016;7:13129.

63. Coward WR, Marei A, Yang A, et al. Statin-induced proinflammatory response in mitogenactivated peripheral blood mononuclear cells through the activation of caspase-1 and IL-18 secretion in monocytes. J Immunol 2006;176:5284-92.

64. Bellosta S, Corsini A. Statin drug interactions and related adverse reactions. Expert Opin Drug Saf 2012;11:933-46.

65. Attout H, Guez S, Ranaivo I, et al. A patient with hyper-lgD syndrome responding to simvastatin treatment. Eur J Intern Med 2008;19:e82-3.

66. Kuijk LM, Mandey SH, Schellens I, et al. Statin synergizes with LPS to induce IL-1beta release by THP-1 cells through activation of caspase-1. Mol Immunol 2008;45:2158-65.

67. Simon A, Drewe E, van der Meer JW, et al. Simvastatin treatment for inflammatory attacks of the hyperimmunoglobulinemia D and periodic fever syndrome. Clin Pharmacol Ther 2004;75:47683.

68. Houten SM, Frenkel J, Rijkers GT, et al. Temperature dependence of mutant mevalonate kinase activity as a pathogenic factor in hyper-lgD and periodic fever syndrome. Hum Mol Genet 2002;11:3115-24.

69. Zanin V, Marcuzzi A, Kleiner G, et al. Lovastatin dose-dependently potentiates the proinflammatory activity of lipopolysaccharide both in vitro and in vivo. J Cardiovasc Transl Res. 2013;6:981-8

70. Thurnher M, Gruenbacher G, Nussbaumer O. Regulation of mevalonate metabolism in cancer and immune cells. Biochim Biophys Acta 2013;1831:1009-15.

71. Diel IJ, Bergner R, Grötz KA. Adverse effects of bisphosphonates: current issues. J Support Oncol 2007 2007;5:475-82.

72. Agrawal AG, Somani RR. Farnesyltransferase inhibitor as anticancer agent. Mini Rev Med Chem 2009;9:638-52.

73. Adami S, Bhalla AK, Dorizzi R, et al. The acute-phase response after bisphosphonate administration. Calcif Tissue Int 1987;41:326-31.

74. Corrado A, Cantatore FP. The bisphosponates: chemical characteristics, skeletal biological effects and extra-skeletal effects. Reumatismo 2005 2005;57:142-53.

75. Makkonen N, Salminen A, Rogers MJ, et al. Contrasting effects of alendronate and clodronate on RAW 264 macrophages: the role of a bisphosphonate metabolite. Eur J Pharm Sci 1999;8:109-18. 
76. Maruyama N, Takizawa $\mathrm{T}$, Ishibashi $\mathrm{H}$, et al. Protective activity of geranium oil and its component, geraniol, in combination with vaginal washing against vaginal candidiasis in mice. Biol Pharm Bull 2008;31:1501-6.

77. Ong TP, Heidor R, de Conti A, et al. Farnesol and geraniol chemopreventive activities during the initial phases of hepatocarcinogenesis involve similar actions on cell proliferation and DNA damage, but distinct actions on apoptosis, plasma cholesterol and HMGCoA reductase. Carcinogenesis 2006;27:1194-203.

78. Chang FY, Lu CL. Treatment of irritable bowel syndrome using complementary and alternative medicine. J Chin Med Assoc 2009;72:294-300.

79. Merat S, Khalili S, Mostajabi $P$, et al. The effect of enteric-coated, delayed-release peppermint oil on irritable bowel syndrome. Dig Dis Sci 2010;55:1385-90.

80. de Moura Espíndola R, Mazzantini RP, Ong TP, et al. Geranylgeraniol and beta-ionone inhibit hepatic preneoplastic lesions, cell proliferation, total plasma cholesterol and DNA damage during the initial phases of hepatocarcinogenesis, but only the former inhibits NF-kappaB activation. Carcinogenesis 2005;26:1091-9.

81. Peterson YK, Kelly P, Weinbaum CA, Casey PJ. A novel protein geranylgeranyltransferase-I inhibitor with high potency, selectivity, and cellular activity. J Biol Chem 2006,281:12445-50.

82. Lackner MR, Kindt RM, Carroll PM, et al. Chemical genetics identifies Rab geranylgeranyl transferase as an apoptotic target of farnesyl transferase inhibitors. Cancer Cell 2005;7:325-36.

83. Zhang FL, Fu HW, Casey PJ, Bishop WR. Substitution of cadmium for zinc in farnesyl:protein transferase alters its substrate specificity. Biochemistry 1996;35:8166-71.

84. Ichikawa M, Ohtsuka M, Ohki H, et al. Discovery of DF-461, a Potent Squalene Synthase Inhibitor. ACS Med Chem Lett 2013;4:932-6.

85. Wierzbicki AS, Hardman TC, Viljoen A. New lipid-lowering drugs: an update. Int J Clin Pract 2012;66:270-80.

86. Bergstrom JD, Kurtz MM, Rew DJ, et al. Zaragozic acids: a family of fungal metabolites that are picomolar competitive inhibitors of squalene synthase. Proc Natl Acad Sci U S A 1993;90:80-4.

87. Bodar EJ, Kuijk LM, Drenth JP, et al. On-demand anakinra treatment is effective in mevalonate kinase deficiency. Ann Rheum Dis 2011;70:2155-8.

88. Vitale A, Rigante D, Lucherini OM, et al. Biological treatments: new weapons in the management of monogenic autoinflammatory disorders. Mediators Inflamm 2013;2013:939847.

89. Arkwright PD, Abinun M, Cant AJ. Mevalonic aciduria cured by bone marrow transplantation. N Engl J Med 2007;357:1350.

90. Neven B, Valayannopoulos V, Quartier P, et al. Allogeneic bone marrow transplantation in mevalonic aciduria. N Engl J Med 2007;356:2700-3.

91. El-Sayyad HI. Cholesterol overload impairing cerebellar function: the promise of natural products. Nutrition 2015;31:621-30.

92. Lane KT, Beese LS. Thematic review series: lipid posttranslational modifications. Structural biology of protein farnesyltransferase and geranylgeranyltransferase type I. J Lipid Res 2006;47:681-99.

93. Suzuki N, Ito T, Matsui H, Takizawa M. Anti-inflammatory and cytoprotective effects of a squalene synthase inhibitor, TAK-475 active metabolite-I, in immune cells simulating mevalonate kinase deficiency (MKD)-like condition. Springerplus 2016;5:1429.

94. Ebihara T, Takeuchi T, Moriya $\mathrm{Y}$, et al. Characterization of Transporters in the Hepatic Uptake of TAK-475 M-I, a Squalene Synthase Inhibitor, in Rats and Humans. Drug Res (Stuttg) 2016;66:316-23.

95. Stein EA, Bays H, O'Brien D, et al. Lapaquistat acetate: development of a squalene synthase inhibitor for the treatment of hypercholesterolemia. Circulation 2011;123:1974-85. 
96. Liao JK. Squalene synthase inhibitor lapaquistat acetate: could anything be better than statins? Circulation 2011;123:1925-8.

97. Seiki S, Frishman WH. Pharmacologic inhibition of squalene synthase and other downstream enzymes of the cholesterol synthesis pathway: a new therapeutic approach to treatment of hypercholesterolemia. Cardiol Rev 2009;17:70-6.

98. Davidson $\mathrm{MH}$. Novel nonstatin strategies to lower low-density lipoprotein cholesterol. Curr Atheroscler Rep 2009;11:67-70.

99. Cochemé HM, Kelso GF, James AM, et al. Mitochondrial targeting of quinones: therapeutic implications. Mitochondrion 2007;7 Suppl:S94-102.

100. Dhanasekaran A, Kotamraju S, Kalivendi SV, et al. Supplementation of endothelial cells with mitochondria-targeted antioxidants inhibit peroxide-induced mitochondrial iron uptake, oxidative damage, and apoptosis. J Biol Chem 2004;279:37575-87. 


\section{TABLES LEGENDS}

Table 1. Mevalonate kinase deficiency clinical phenotype based on the typical signs and symptoms observed in the patients.

\section{FIGURES LEGENDS}

\section{Figure 1.}

Pathogenesis of inflammatory processes in Mevalonate Kinase Deficiency (MKD). BisP:

bisphosphonates; $\mathrm{CH} 25 \mathrm{H}$ : cholesterol 25-hydroxylase; FTI: farnesyltransferase inhibitors; GGTI: geranylgeranyl transferase inhibitors; HMGCR: 3-hydroxy-3-methylglutaryl-CoA reductase; MK: mevalonate kinase; S Synth: squalene synthase; ZAA: zaragozig acid.

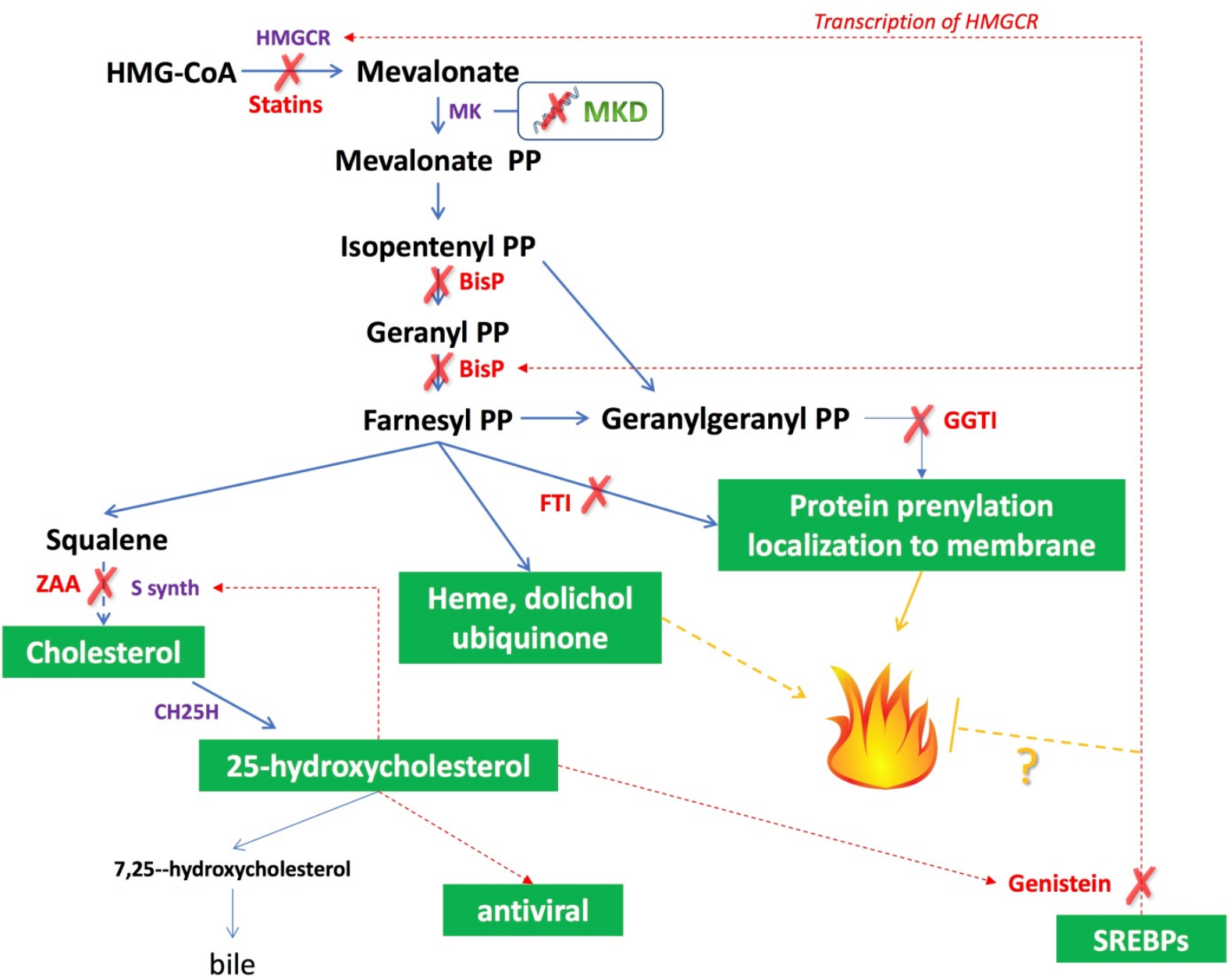


Figure 2.

Therapeutic options in Mevalonate Kinase Deficiency. 


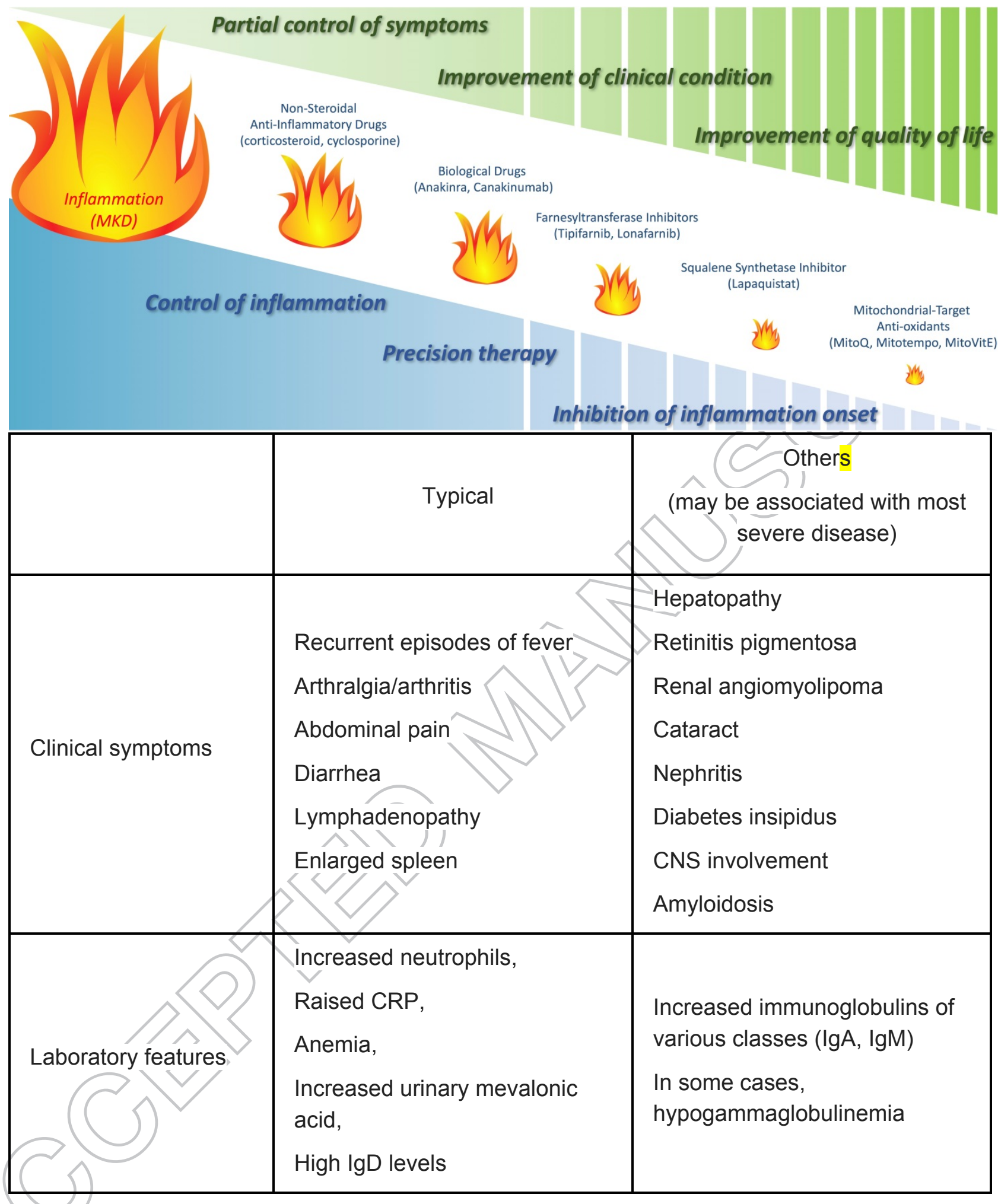

Table 1. Mevalonate kinase deficiency clinical phenotypes based on the typical signs and symptoms observed in the patients. 